\title{
Regularity of invariant graphs over hyperbolic systems
}

\author{
D. HADJILOUCAS $\dagger$, M. J. NICOL $\ddagger$ and C. P. WALKDEN§ \\ $\dagger$ Department of Mathematics, UMIST, Manchester M60 1QD, UK \\ (e-mail: demetris.hadjiloucas@umist.ac.uk) \\ $\ddagger$ Department of Mathematics and Statistics, University of Surrey, Guildford, \\ Surrey GU2 7XH, UK \\ (e-mail:m.nicol@surrey.ac.uk) \\ $\S$ Department of Mathematics, Manchester University, Oxford Road, \\ Manchester M13 9PL, UK \\ (e-mail: cwalkden@ma.man.ac.uk)
}

(Received 19 October 1999 and accepted in revised form 7 February 2001)

\begin{abstract}
We consider cocycles with negative Lyapunov exponents defined over a hyperbolic dynamical system. It is well known that such systems possess invariant graphs and that under spectral assumptions these graphs have some degree of Hölder regularity. When the invariant graph has a slightly higher Hölder exponent than the a priori lower bound on an open set (even on just a set of positive measure for certain systems), we show that the graph must be Lipschitz or (in the Anosov case) as smooth as the cocycle.
\end{abstract}

\section{Introduction}

In this paper we consider the regularity of dynamically invariant graphs of skew-product systems with uniformly hyperbolic base dynamics and uniform or non-uniform contraction in the fibre.

Our work is related to that of Hirsch et al [2], Stark [8, 9] and Kaplan et al [4], who have investigated the existence and regularity properties of invariant graphs in skew-product systems. Similar results have been proved in the setting of group-valued cocycle equations $[5,6]$.

Suppose $f: M \rightarrow M$ is a $C^{1}$ diffeomorphism of a $C^{\infty}$ compact Riemannian manifold $M$ and suppose that $\Lambda$ is a locally maximal hyperbolic set and $\left.f\right|_{\Lambda}$ is an Axiom A diffeomorphism which has an invariant equilibrium measure $m$ corresponding to a Hölder continuous function. Often we shall assume that $\Lambda=M$, so that $f$ is Anosov.

There exist constants $C>0, \mu_{s, \max } \geq \mu_{s}>0$ and $\mu_{u, \max } \geq \mu_{u}>0$ such that on local stable manifolds $\left\{W_{\text {loc }}^{s}(x)\right\}$,

$$
C e^{-n \mu_{s, \max }} d(x, y) \leq d\left(f^{n} x, f^{n} y\right) \leq C e^{-n \mu_{s}} d(x, y)
$$


for $y \in W_{\mathrm{loc}}^{s}(x)$ and

$$
C e^{-n \mu_{u, \max }} d(x, y) \leq d\left(f^{-n} x, f^{-n} y\right) \leq C e^{-n \mu_{u}} d(x, y)
$$

for $y \in W_{\mathrm{loc}}^{u}(x)$, the local unstable manifold. Thus $\mu_{s, \max }$ and $\mu_{s}$ correspond to the maximum and minimum rates of contraction along stable manifolds, respectively. We denote the conditional measures of $m$ along the stable $\left(\left\{W_{\text {loc }}^{s}(x)\right\}\right)$ and unstable $\left(\left\{W_{\mathrm{loc}}^{u}(x)\right\}\right)$ foliations by $m_{s}(x)$ and $m_{u}(x)$ respectively. We will write $m_{s}, m_{u}$ when the point $x$ is clear by context.

Let $Y$ be a complete separable metric space and suppose that $g: \Lambda \times Y \rightarrow Y$ is a continuous mapping which is $\alpha$-Hölder in the first component; that is, for each compact $W \subset Y$ there exists a $K_{W}$ such that

$$
d\left(g(x, y), g\left(x^{\prime}, y\right)\right) \leq K_{W} d\left(x, x^{\prime}\right)^{\alpha}
$$

for each $y \in W$. We shall often assume that $g$ is $C^{k}$; in this case, we implicitly require $Y$ to have a smooth Riemannian structure.

We form the skew-product

$$
F(x, y)=(f(x), g(x, y)) .
$$

To simplify notation we write $g(x, y)=g(x) y$ and consider $g: \Lambda \rightarrow C(Y, Y)$ as a family of continuous mappings of $Y$. If for each $x, g(x)$ is assumed invertible (as we will for most of this paper) we denote $g(x)^{-1}$ by $h(x)$. We define $g^{n}(x)$ by $F^{n}(x, y)=\left(f^{n}(x), g^{n}(x) y\right)$ and similarly define (where appropriate) $h^{n}(x)$ by $F^{-n}(x, y)=\left(f^{-n}(x), h^{n}(x) y\right)$. For heuristic reasons we also write $g^{n}(x) y$ as $g\left(f^{n-1} x\right) g\left(f^{n-2} x\right) \cdots g(x) y$, i.e. as a composition of operators acting on $Y$.

We say that $g$ is uniformly contracting if there exists $\lambda_{u}<0$ and $c>0$ such that

$$
d\left(g^{n}(x) y, g^{n}(x) y^{\prime}\right) \leq c e^{n \lambda_{u}} d\left(y, y^{\prime}\right)
$$

for all $x \in X$. Take any continuous function $v: \Lambda \rightarrow Y$. Then it is easy to check that $g^{n}\left(f^{-n} x\right) v\left(f^{-n} x\right)$ converges uniformly to a continuous function $\phi$. Moreover, the graph of $\phi$ is invariant under the skew product $F$; equivalently $\phi$ solves the functional equation

$$
\phi(f x)=g(x) \phi(x) .
$$

Moreover, $\phi$ is unique.

Under the additional assumption that $g$ is $\alpha$-Hölder for some $0<\alpha \leq 1$ then the following result provides a degree of regularity for $\phi$.

Proposition 1.1. [2, 8, 9] Suppose $g$ is $\alpha$-Hölder in the first coordinate. Then $\phi$ is uniformly Hölder on $\Lambda$ with exponent $\gamma$, for any $0<\gamma \leq \alpha$, such that $\gamma<-\lambda_{u} / \mu_{s, \max }$. In particular if $\lambda_{u}<-\mu_{s, \max }$ and $\alpha=1$, then $\phi$ is Lipschitz.

Remark 1.2. Proposition 1.1 does not need the hyperbolicity assumption on $f$; we merely need to know the maximum amount of expansion by $f^{-1}$.

Assume that $\lambda_{s} \in\left(-\infty, \lambda_{u}\right)$ is the maximum rate of contraction for $g$, i.e. there exists $C>0$ such that for all $n \in \mathbb{N}$

$$
C e^{n \lambda_{s}} d\left(y, y^{\prime}\right) \leq d\left(g^{n}(x) y, g^{n}(x) y^{\prime}\right) .
$$


(Note that this implies $g(x)$ is invertible for each $x$.) The map $F$ is said to be partially hyperbolic if

$$
-\mu_{s}<\lambda_{s} \leq \lambda_{u}<\mu_{u}
$$

We will assume from now on that our skew-product is partially hyperbolic. In the case of uniform contraction the system satisfies

$$
-\mu_{s}<\lambda_{s} \leq \lambda_{u}<0<\mu_{u}
$$

The main result of this paper states that in our set-up Proposition 1.1 is generically optimal. To be more precise we introduce some useful definitions.

We say that $\phi$ restricted to a set $S,\left.\phi\right|_{S}$, is Hölder of exponent $\alpha$ if there exists a constant $C>0$ such that $d(\phi(x), \phi(y)) \leq C d(x, y)^{\alpha}$ for all $x, y \in S$.

A map $f: M \rightarrow M$ is said to be conformal if there exists a scalar function $a(x)$ such that $d_{x}^{s} f=a(x) \operatorname{Isom}(x)$ where $\operatorname{Isom}(x)$ is an isometry.

The hyperbolic map $f$ is said to be $s$-conformal if the derivative $d^{s} f$ of $f$ in the stable direction is conformal. We are now ready to state our main results in the case of uniform contraction. We assume that $F(x, y)=(f(x), g(x) y)$ is a partially hyperbolic system as previously described. We say that a function $\psi$ is $C^{k-}$ if it is $C^{k-1}$ and the $(k-1)^{\text {st }}$ derivative is $C^{\gamma}$ for any $\gamma \in(0,1)$ (with the conventions that $C^{1-}=C^{1}$ and $C^{\infty-}=C^{\infty}$ ).

THEOREM 1.3. Let $f: M \rightarrow M$ be a $C^{k}$ Anosov diffeomorphism of a $C^{\infty}$ compact Riemannian manifold $M$. Suppose that the $C^{k} \operatorname{map}(x, y) \mapsto g(x, y)$ is uniformly contracting and $F(x, y)=(f(x), g(x) y): M \times Y \mapsto M \times Y$ is partially hyperbolic. Suppose that $\phi$ determines the invariant graph, i.e. $\phi$ is the continuous solution to the functional equation

$$
g(x) \phi(x)=\phi(f x) .
$$

Then for any $0<\gamma \leq 1$ such that $\gamma>-\lambda_{s} / \mu_{s}$ either:

(i) $\phi$ is not $C^{\gamma}$ on any open set $S$; or

(ii) $\phi$ is $C^{k-}$.

If $f$ is s-conformal then:

(a) $\left.\phi\right|_{S}$ is not $C^{\gamma}$ on any set $S$ of positive measure; or

(b) $\phi$ is $C^{k-}$.

Moreover, generically (in the space of $C^{k}$ partially hyperbolic uniformly contracting cocycles $g),(i),(a)$ occur.

Remark 1.4. Under the conditions of the theorem, the graph of $\phi$ when restricted to local unstable manifolds is always $C^{k}$. Note also that $\phi$ is $\gamma^{\prime}$-Hölder for any $\gamma^{\prime}$ satisfying $\gamma^{\prime}<-\lambda_{u} / \mu_{s, \max }$.

Remark 1.5. A similar result is proved in [1]. They consider the regularity of the subbundles $E^{s}, E^{u}$ in the Anosov splitting of the tangent bundle of $M$. These sub-bundles are always Hölder and, generically, this degree of regularity is nowhere exceeded.

Remark 1.6. A similar result holds if we assume only that $f$ is Axiom A. Specifically, if $F$ is partially hyperbolic and, for each $x \in \Lambda, g(x)$ is bi-Lipschitz then the analogue of Theorem 1.3 holds if property (ii) states that $\phi$ is Lipschitz. 
Remark 1.7. The dichotomy between (i) and (ii) of Theorem 1.3 holds under the hypotheses that $\phi$ is continuous and the cocycle $g$ is partially hyperbolic. The uniform contraction assumption is only required to ensure that the graph $\phi$ is continuous.

Remark 1.8. The $s$-conformal hypothesis is a technical assumption used to avoid a lengthy digression in the proofs.

We remark that Kaplan et al [4] consider a family of skew-products over hyperbolic toral automorphisms with affine cocycles and have results on the regularity and boxcounting dimension $\operatorname{dim}_{B}(\operatorname{graph}(\phi))$ of the invariant graph. Specifically, they study the skew-product $F: T^{k} \times \mathbb{R} \rightarrow T^{k} \times \mathbb{R}:(x, y) \mapsto(A x, p(x)+\lambda y)$ where $T^{k}$ denotes the $k$-dimensional torus, $A: T^{k} \rightarrow T^{k}$ is a linear hyperbolic toral automorphism, $\lambda \in \mathbb{R}$ and $p: T^{k} \rightarrow \mathbb{R}$ is a sufficiently smooth function of period 1 in each coordinate. When $k=2, A$ has eigenvalues $\mu, \mu^{-1}$ with $|\mu|<1$. Provided that $\mu<\lambda<1$, the system is uniformly contracting and partially hyperbolic so that the attracting invariant graph is continuous, indeed Hölder. The following dichotomy holds.

Proposition 1.9. [4] Let $A$ be a hyperbolic automorphism of $T^{2}$ with eigenvalues $\mu, \mu^{-1},|\mu|<1$. Let $p: T^{2} \rightarrow \mathbb{R}$ be $C^{3}$ and suppose that $\lambda \in(\mu, 1)$. Then either:

(i) $\phi$ is nowhere differentiable and $\operatorname{dim}_{B}(\operatorname{graph}(\phi))=3-(\log \lambda / \log \mu)$; or

(ii) $\phi$ is smooth (in which case $\operatorname{dim}_{B}(\operatorname{graph}(\phi))=2$ ).

Dimension estimates in our set-up will appear in a forthcoming paper [10].

1.1. Non-uniform contraction. We now discuss our results in the context in which $g$ contracts non-uniformly. We still assume that there exist constants $C, c>0, \lambda_{s} \leq \lambda_{u}$, such that for all $n \in \mathbb{N}$ equations (1.2) and (1.4) hold but $\lambda_{u}>0$ is allowed. In this setting we are not guaranteed the existence of a continuous invariant graph (or indeed an invariant graph at all) and so we will consider the regularity properties of invariant measurable graphs.

One context in which there always exists a measurable attracting invariant graph is that of 'contraction on average'.

Suppose that

$$
\rho(x)=\lim _{n \rightarrow \infty} \sup _{y, y^{\prime} \in Y} \frac{1}{n} \log \frac{d\left(g^{n}(x) y, g^{n}(x) y^{\prime}\right)}{d\left(y, y^{\prime}\right)}
$$

exists for $m$-a.e. $x \in \Lambda$. (This is the maximal Lyapunov exponent in the fibre direction of the skew product $F$.) This condition is satisfied if, for example, the functions $\log ^{+}\|g(x)\|, \log ^{+}\left\|g(x)^{-1}\right\| \in L^{1}(\Lambda, m)$ where

$$
\|g(x)\|:=\sup _{y, y^{\prime} \in Y} \frac{d\left(g(x) y, g(x) y^{\prime}\right)}{d\left(y, y^{\prime}\right)}
$$

(and $\left\|g(x)^{-1}\right\|$ is defined similarly). In this context we will say that the skew-product contracts on average if there exists $\rho<0$ such that

$$
\rho(x) \leq \rho<0
$$

for $m$-a.e. $x \in \Lambda$. As a consequence of contraction on average we have the following result of Stark [9], which applies in a more general setting. 
Proposition 1.10. [9] Suppose $X$ is a compact metric space, $f: X \rightarrow X a$ homeomorphism, $m$ an $f$-invariant measure, $Y$ a complete metric space and $g: X \times$ $Y \rightarrow Y$ is continuous and satisfies (1.7). Then there exists an $f$-invariant set $\Lambda \subset X$ such that $m(\Lambda)=1$ and a measurable function $\phi: \Lambda \rightarrow Y$ such that the graph of $\phi$ is invariant and attracting under $(f, g)$.

Note that in this case the graph $\phi: \Lambda \rightarrow Y$ is measurable and satisfies the functional equation (1.3) for $m$-a.e. $x \in X$.

Contraction on average implies that for $m$-a.e. $x \in \Lambda$, given $\epsilon>0$ there exists a measurable function $c: \Lambda \rightarrow \mathbb{R}$ such that

$$
d\left(g^{n}(x) y, g^{n}(x) y^{\prime}\right) \leq c(x) e^{n(\rho+\epsilon)} d\left(y, y^{\prime}\right) .
$$

Stark has the following regularity results in the contraction on average setting.

Proposition 1.11. [9] Suppose further that $f$ is a $C^{1+\alpha}$ diffeomorphism, $\rho<0$, $c(x) \in L^{1}(\Lambda, m)$ and for a point $y_{0} \in Y$ there exist constants $\beta, K_{1}, K_{2}$ such that

$$
d\left(g(x) y, g\left(x^{\prime}\right) y\right) \leq\left(K_{1}+K_{2} d\left(y, y_{0}\right)^{\beta}\right) d\left(x, x^{\prime}\right)^{\alpha} .
$$

Then for any $0<\gamma \leq \alpha$ such that $\gamma<-\rho / \mu_{s, \max }$ we can construct a nested sequence $\Lambda_{k}, k \in \mathbb{N}$ of compact sets such that $m\left(\Lambda_{k}\right) \rightarrow 1$ and $\left.\phi\right|_{\Lambda_{k}}$ is Hölder of exponent $\gamma$.

We now assume that $f$ is a $C^{k}$ Anosov diffeomorphism, equipped with an ergodic equilibrium measure $m$ corresponding to a Hölder function and that $g(x)$ is invertible for each $x$ (denote $\left.h(x)=g(x)^{-1}\right)$. We will say that $g$ is centre bunched if $\lambda_{u}-\lambda_{s}<$ $\min \left\{\mu_{s}, \mu_{u}\right\}$ (cf. [6]).

We will always assume that in the case of non-uniform contraction we are (roughly) in the setting of Proposition 1.11. More precisely we assume that for a point $y_{0} \in Y$ there exist constants $K_{1}, K_{2}$ such that:

(I) $\quad d\left(g(x) y, g\left(x^{\prime}\right) y\right) \leq\left(K_{1}+K_{2} d\left(y, y_{0}\right)\right) d\left(x, x^{\prime}\right)$; and

(II) $\quad d\left(h(x) y, h\left(x^{\prime}\right) y\right) \leq\left(K_{1}+K_{2} d\left(y, y_{0}\right)\right) d\left(x, x^{\prime}\right)$

(we have taken $\alpha=\beta=1$ in Proposition 1.11). Suppose that $\rho<0$ in addition to (I), (II). Let $\phi$ be the unique measurable invariant graph for the partially hyperbolic map $F$ which satisfies (I), (II) and $\rho<0$. Under the assumption of (I), (II) and $\rho<0$ we have the following generalization of Theorem 1.3 in the non-uniform case.

THEOREM 1.12. Suppose that the $C^{k}$ map $g$ is centre bunched, non-uniformly contracting $(\rho<0)$ and satisfies $(I)$, (II). Then for any $0<\gamma \leq 1$ such that $\gamma>-\lambda_{s} / \mu_{s}$ either:

(i) $\left.\phi\right|_{S}$ is not $C^{\gamma}$ on any open set $S$; or

(ii) $\phi$ is $C^{k-}$.

If $f$ is s-conformal then either:

(a) $\left.\phi\right|_{S}$ is not $C^{\gamma}$ on any set $S$ of positive measure; or

(b) $\phi$ is $C^{k-}$.

Moreover, generically (i), (a) occur.

Remark 1.13. If we assume that $f: \Lambda \rightarrow \Lambda$ is Axiom A rather than Anosov, then the conclusions of Theorem 1.12 hold if properties (ii), (b) state that $\phi$ is Lipschitz. 
Remark 1.14. Consider the case when $\phi$ is assumed to be an invariant measurable graph for the partially hyperbolic mapping $F$ satisfying conditions (I) and (II) (i.e. we make no assumption of contraction on average) and the base is assumed to be an ergodic (with respect to a Hölder equilibrium measure $m$ ) Anosov diffeomorphism. Then we have the following regularity results.

THEOREM 1.15. Suppose that the $C^{k}$ map $g$ is centre bunched, satisfies (I), (II) and $\phi$ is measurable. Then for any $0<\gamma \leq 1$ such that $\gamma>\max \left\{-\lambda_{s} / \mu_{s}, \lambda_{u} / \mu_{u}\right\}$ either:

(i) $\left.\phi\right|_{S}$ is not $C^{\gamma}$ on any open set $S$; or

(ii) $\phi$ is $C^{k-}$.

Moreover, generically $(i)$ occurs.

Remark 1.16. If we assume that $f: \Lambda \rightarrow \Lambda$ is Axiom A rather than Anosov and $g(x)$ is bi-Lipschitz, then the conclusions of Theorem 1.15 hold if property (ii) states that $\phi$ is Lipschitz.

Remark 1.17. We do not know if the centre bunching condition $\left(\lambda_{u}-\lambda_{s}<\min \left\{\mu_{u}, \mu_{s}\right\}\right)$ in the statements of Theorem 1.12 and Theorem 1.15 can be relaxed to merely the partial hyperbolicity condition $-\mu_{s}<\lambda_{s}<\lambda_{u}<\mu_{u}$ of the uniform case (Theorem 1.3).

\section{Proof of Theorem 1.3}

We discuss the regularity of $\phi$ along the stable and unstable manifolds of $f$ independently.

2.1. Regularity on local stable manifolds. Let $x \in \Lambda$. For each $n>0$, define

$$
\gamma_{x}^{s, n}(\cdot): W_{\mathrm{loc}}^{s}(x) \rightarrow Y: t \mapsto h^{n}(t) \phi\left(f^{n} x\right) .
$$

Then

$$
\begin{aligned}
d\left(\gamma_{x}^{s, n+1}(t), \gamma_{x}^{s, n}(t)\right) & =d\left(h^{n}(t) h\left(f^{n} t\right) g\left(f^{n} x\right) \phi\left(f^{n} x\right), h^{n}(t) h\left(f^{n} t\right) g\left(f^{n} t\right) \phi\left(f^{n} x\right)\right) \\
& \leq C e^{-n \lambda_{s}} d\left(g\left(f^{n} x\right) \phi\left(f^{n} x\right), g\left(f^{n}(t)\right) \phi\left(f^{n} x\right)\right) .
\end{aligned}
$$

As $\phi$ is continuous, hence bounded, we have

$$
\begin{aligned}
d\left(g\left(f^{n} x\right) \phi\left(f^{n} x\right), g\left(f^{n} t\right) \phi\left(f^{n} x\right)\right) & \leq K d\left(f^{n} x, f^{n} t\right)^{\alpha} \\
& \leq K e^{-n \alpha \mu_{s}} d(x, t)^{\alpha}
\end{aligned}
$$

where $\alpha$ is the Hölder constant of $x \mapsto g(x, \cdot)$. Hence

$$
d\left(\gamma_{x}^{s, n+1}(t), \gamma_{x}^{s, n}(t)\right) \leq C e^{-n\left(\lambda_{s}+\alpha \mu_{s}\right)} d(x, t)^{\alpha}
$$

for some constant $C>0$, independent of $x, t$ and $n$. Hence if $\alpha>-\lambda_{s} / \mu_{s},\left\{\gamma_{x}^{s, n}(t)\right\}$ is Cauchy by the partial hyperbolicity assumption and therefore converges. Denote the limit by $\gamma_{x}^{s}(t)$.

LEMMA 2.1. The graph of $\gamma_{x}^{s}(\cdot)$ is $F$-invariant in the sense that $F\left(t, \gamma_{x}^{s}(t)\right)=(f(t)$, $\left.\gamma_{f(x)}^{s}(f t)\right)$. 
Proof. As $F\left(t, \gamma_{x}^{s}(t)\right)=\left(f(t), g(t) \gamma_{x}^{s}(t)\right)$ the claim follows from the fact that

$$
\begin{aligned}
g(t) \gamma_{x}^{s}(t) & =\lim _{n \rightarrow \infty} g(t) h^{n}(t) g^{n}(x) \phi(x) \\
& =\lim _{n \rightarrow \infty} g(t) h(t) h^{n-1}(f t) g^{n-1}(f x) g(x) \phi(x) \\
& =\gamma_{f x}^{s}(f t) .
\end{aligned}
$$

The following quantity shall prove useful. For $t \in W_{\text {loc }}^{s}(x)$, define

$$
s_{j}(t)=d\left(h(t) \cdots h\left(f^{j-1} t\right) h\left(f^{j} t\right) \phi\left(f^{j} x\right), h(t) \cdots h\left(f^{j-1} t\right) h\left(f^{j} x\right) \phi\left(f^{j} x\right)\right) .
$$

Lemma 2.2. The maps $t \mapsto \gamma_{x}^{s}(t): W_{\mathrm{loc}}^{s}(x) \rightarrow Y$ are uniformly Lipschitz.

Proof. Observe that

$$
\begin{aligned}
d\left(\gamma_{x}^{s, n}(t), \gamma_{x}^{s, n}(x)\right) & =d\left(h^{n}(t) \phi\left(f^{n} x\right), h^{n}(x) \phi\left(f^{n} x\right)\right) \\
& \leq \sum_{j=0}^{n-1} d\left(h(t) \cdots h\left(f^{j-1} t\right) h\left(f^{j} t\right) h\left(f^{j+1} x\right) \cdots h\left(f^{n-1} x\right) \phi\left(f^{n} x\right)\right. \\
& \left.h(t) \cdots h\left(f^{j-1} t\right) h\left(f^{j} x\right) h\left(f^{j+1} x\right) \cdots h\left(f^{n-1} x\right) \phi\left(f^{n} x\right)\right) \\
& =\sum_{j=0}^{n-1} s_{j}(t) .
\end{aligned}
$$

We estimate

$$
\begin{aligned}
s_{j}(t) & \leq C e^{-\lambda_{s} j} d\left(h\left(f^{j} t\right) \phi\left(f^{j} x\right), h\left(f^{j} x\right) \phi\left(f^{j} x\right)\right) \\
& \leq C e^{-\lambda_{s} j} d\left(f^{j} t, f^{j} x\right) \\
& \leq C e^{-j\left(\lambda_{s}+\mu_{s}\right)} d(t, x) .
\end{aligned}
$$

By the partial hyperbolicity assumption, we see that

$$
d\left(\gamma_{x}^{s, n}(t), \gamma_{x}^{s, n}(x)\right) \leq \sum_{j=0}^{n-1} s_{j}(t) \leq \sum_{j=0}^{\infty} s_{j}(t) \leq C d(t, x)
$$

Letting $n \rightarrow \infty$ gives the result.

For each $x \in \Lambda$ and $t \in W_{\text {loc }}^{s}(x)$ define

$$
\begin{aligned}
\Delta(x, t) & =d\left(\phi(t), \gamma_{x}^{s}(t)\right) \\
\Delta(x) & =\sup _{t \in W_{\mathrm{loc}}^{s}(x)} \Delta(x, t) .
\end{aligned}
$$

The functions $\Delta(x, t)$ and $\Delta(x)$ will act as obstructions to the regularity of $\phi$ along the local stable manifold through $x$. Note that $x \mapsto \Delta(x)$ is continuous.

LEMMA 2.3. If $\phi$ is $C^{\gamma}$ on an open set $S$, where $\gamma>-\lambda_{s} / \mu_{s}$, then $\phi$ is globally uniformly Lipschitz on local stable manifolds. 
Proof. By Poincaré recurrence, almost every point of $\Lambda$ returns to $S$ infinitely often. Hence there exists a sequence $n_{j} \uparrow \infty$ such that $f^{n_{j}} x, f^{n_{j}} t \in S$. Then for each $n_{j}$

$$
\begin{aligned}
d\left(\phi(t), \gamma_{x}^{s}(t)\right) & =d\left(h^{n_{j}}(t) \phi\left(f^{n_{j}} t\right), h^{n_{j}}(t) \phi\left(f^{n_{j}} x\right)\right) \\
& \leq C e^{-n_{j}\left(\lambda_{s}+\gamma \mu_{s}\right)} d(x, t)^{\gamma} .
\end{aligned}
$$

Letting $n_{j} \rightarrow \infty$ yields $\Delta(x)=0$ by partial hyperbolicity and the assumption on $\gamma$. Similarly $\Delta\left(f^{n} x\right)=0$ for each $n>0$ so that $\Delta(x)=0$ on a dense set; hence $\Delta(x)=0$ everywhere and the lemma follows.

If $f$ is $s$-conformal then we may improve this lemma in the following way.

LEMMA 2.4. If $\left.\phi\right|_{S}$ is $C^{\gamma}$, where $\gamma>-\lambda_{s} / \mu_{s}$ and $m(S)>0$, then $\phi$ is globally uniformly Lipschitz on local stable manifolds.

Proof. By Lemma A.1 of Appendix A we may assume that there exists a sequence $n_{j} \uparrow \infty$ such that $f^{n_{j}} x, f^{n_{j}} t \in S$ and the proof follows as in Lemma 2.3.

We now show that if $\phi$ is globally Lipschitz then it is as smooth as $g$ along the local stable manifold through $x \in \Lambda$. Note that by Lemma 2.4, if the graph of $\phi$ is Lipschitz then the graph of $\phi$ contains the graphs of the functions $\gamma_{x}^{s}$. To show that $\phi$ is $C^{k}$ along local stable manifolds, it suffices to prove that the functions $\gamma_{x}^{s}$ are $C^{k}$. To do this, we show that the graphs of the functions $\gamma_{x}^{s}$ are the local stable manifolds for the partially hyperbolic (in the sense of [2]) skew product $F$. The local stable manifolds of $F$ are as smooth as $g$. So by [2, Theorem 5.5, p. 61], it is sufficient to show that the rate of convergence of orbits along the graph of $\gamma_{x}^{s}$ is exponential at rate $-\mu_{s}$ (possibly replacing $-\mu_{s}$ by $-\mu_{s}+\epsilon$ for a suitably small $\epsilon>0$ ).

LEMMA 2.5. There exists a constant $C>0$ such that for all $x \in \Lambda$ and $t \in W_{\mathrm{loc}}^{s}(x)$, we have

$$
d\left(F^{n}\left(t, \gamma_{x}^{s}(t)\right), F^{n}(x, \phi(x))\right) \leq C e^{-n \mu_{s}} .
$$

Proof. Note that $F^{n}\left(t, \gamma_{x}^{s}(t)\right)=\left(f^{n}(t), g^{n}(t) \gamma_{x}^{s}(t)\right)$ and $F^{n}(x, \phi(x))=\left(f^{n}(x)\right.$, $\left.\phi\left(f^{n}(x)\right)\right)$. As $d\left(f^{n}(t), f^{n}(x)\right) \leq C e^{-n \mu_{s}} d(x, t)$, it suffices to prove that $d\left(g^{n}(t) \gamma_{x}^{s}(t), \phi\left(f^{n}(x)\right)\right) \leq C e^{-n \mu_{s}} d(x, t)$.

Now

$$
\begin{aligned}
d\left(g^{n}(t) \gamma_{x}^{s}(t), \phi\left(f^{n} x\right)\right) & =d\left(g^{n}(t) h^{n+m}(t) \phi\left(f^{n+m} x\right), h^{m}\left(f^{n} x\right) \phi\left(f^{n+m} x\right)\right) \\
& =d\left(h^{m}\left(f^{n} t\right) \phi\left(f^{n+m} x\right), h^{m}\left(f^{n} x\right) \phi\left(f^{n+m} x\right)\right) \\
& \leq \sum_{j=0}^{n-1} s_{j}\left(f^{n} t\right) .
\end{aligned}
$$

Using similar arguments to those in Lemma 2.2, it is easy to check that

$$
s_{j}\left(f^{n} t\right) \leq C e^{-n \mu_{s}-j\left(\lambda_{s}+\mu_{s}\right)} d(t, x) .
$$


Hence

$$
\sum_{j=0}^{n-1} s_{j, n} \leq C e^{-n \mu_{s}} \sum_{j=0}^{\infty} e^{-j\left(\lambda_{s}+\mu_{s}\right)} d(t, x) .
$$

By partial hyperbolicity, the sum on the right-hand side of (2.12) is finite, and the lemma follows.

2.2. Regularity along unstable manifolds. We show that $\phi$, when restricted to unstable manifolds, is always as regular as $g$. We emphasize that we do not need any partial hyperbolicity assumption here and we remark that the analogues of the functions $\Delta(x, t)$ and $\Delta(x)$ are always identically equal to zero.

LEMMA 2.6. Let $\phi$ be an invariant graph corresponding to a $C^{k}$ cocycle $g$. Then $\phi$ is uniformly $C^{k}$ along each unstable manifold.

Proof. The proof is similar to (but easier than) the previous stable manifold case, so we merely sketch the arguments.

Define for each $x \in \Lambda$ and $n>0$,

$$
\gamma_{x}^{u, n}(\cdot): W_{\mathrm{loc}}^{u}(x) \rightarrow Y: t \mapsto g^{n}\left(f^{-n} t\right) \phi\left(f^{-n} x\right) .
$$

This is easily seen to be Cauchy (and we do not need the partial hyperbolicity assumption for this to hold). Hence the limit $\gamma_{x}^{u}(t)=\lim _{n \rightarrow \infty} \gamma_{x}^{u, n}(t)$ exists.

One can again check that the family of graphs defined by $\gamma_{x}^{u}$ are Lipschitz and are invariant in the sense that $F^{-1}\left(t, \gamma_{x}^{u}(t)\right)=\left(f^{-1}(t), \gamma_{f^{-1} x}^{u}\left(f^{-1} t\right)\right)$. A similar argument to that for the local stable manifold case allows us to conclude that $\gamma_{x}^{u}(t)=\phi(t)$ (without any partial hyperbolicity assumption). Finally, the convergence under $f^{-1}$ in the graphs is exponential at rate $e^{-\mu_{u}}$. Hence, by [2], these graphs correspond to the unstable manifolds of $F$ and are therefore $C^{k}$.

Proof of Theorem 1.3 We have seen that if alternative (i) or (b) in Theorem 1.3 fails then $\phi$ is uniformly $C^{k}$ along local stable and unstable manifolds. By a result of Journé [3], we can conclude that $\phi$ is globally $C^{k-}$.

2.3. Generically the graph is nowhere $C^{\gamma}$. We now show that generically either (i) or (a) occur in Theorem 1.3. If such a set does exist then $\Delta(x, t) \neq 0$ and in fact the function $\Delta(x, t)$ is an obstruction to the regularity of $\phi$ along stable manifolds. Also, recall that along unstable manifolds $\phi$ is always $C^{k}$.

We now show that for a generic set of cocycles $\Delta(x, t) \neq 0$; hence for this set of cocycles $\phi$ is not $C^{\gamma}$ when restricted to a set of positive measure.

Recall that $\phi$ is the unique continuous function $\phi: \Lambda \rightarrow Y$ satisfying $g(x) \phi(x)=$ $\phi(f x)$ for all $x \in \Lambda$. Note that for fixed $v \in Y, \phi(x)=\lim _{n \rightarrow \infty} g\left(f^{-1} x\right) \cdots g\left(f^{-n} x\right) v$ uniformly in $n$.

Thus

$$
\begin{aligned}
\gamma_{x}^{s}(t) & =\lim _{n \rightarrow \infty} h^{n}(t) \phi\left(f^{n} x\right) \\
& =\lim _{n \rightarrow \infty} \lim _{m \rightarrow \infty} g(t)^{-1} \cdots g\left(f^{n-1} t\right)^{-1} g\left(f^{n-1} x\right) \cdots g(x) \cdots g\left(f^{-m} x\right) v .
\end{aligned}
$$


Now choose a periodic point $x$ and a point $t^{\prime} \in W_{\mathrm{loc}}^{s}(x) \cap W_{\mathrm{loc}}^{u}(x)$ homoclinic to $x$. There exists a neighbourhood $U$ of $t^{\prime}$ such that $U$ is disjoint from $f^{n}\left(t^{\prime}\right)$ for $n \in \mathbb{Z} \backslash\{0\}$ and the orbit of $x$.

We perturb $g$ within the class of $C^{k}$ diffeomorphisms (or bi-Lipschitz homeomorphisms in the Axiom A case). Denote the perturbed cocycle by $\tilde{g}$. By making a sufficiently small perturbation, we can assume that $\tilde{g}$ satisfies the partial hyperbolicity conditions. We denote the corresponding perturbed objects by $\tilde{\gamma_{x}^{s}}, \tilde{\phi}$ and $\tilde{\Delta}$.

Since $\tilde{g}\left(f^{-m} t^{\prime}\right)=g\left(f^{-m} t^{\prime}\right)$ for $m \geq 1$, we have $\phi\left(t^{\prime}\right)=\tilde{\phi}\left(t^{\prime}\right)$. Note that $\tilde{\gamma}_{x}^{s}\left(t^{\prime}\right)=$ $\tilde{g}\left(t^{\prime}\right)^{-1} g\left(t^{\prime}\right) \gamma_{x}^{s}\left(t^{\prime}\right)$. Hence

$$
\left|\tilde{\Delta}\left(x, t^{\prime}\right)-\Delta\left(x, t^{\prime}\right)\right|=\left|d\left(\tilde{g}\left(t^{\prime}\right)^{-1} g\left(t^{\prime}\right) \gamma_{x}^{s}\left(t^{\prime}\right), \phi\left(t^{\prime}\right)\right)-d\left(\gamma_{x}^{s}\left(t^{\prime}\right), \phi\left(t^{\prime}\right)\right)\right|,
$$

from which it is easy to see that for a given $t^{\prime}$, there is an open dense set of perturbations $\tilde{g}$ of $g$ for which the right-hand side is not zero, and the result follows.

\section{The case of non-uniform contraction}

The proofs of Theorems 1.12 and 1.15 are similar and differ in detail only. We will show first that under the conditions of Theorem 1.12 if property (i) (or (a) if $f$ is assumed $s$-conformal) does not hold, then the graph of $\phi$ has a Lipschitz version in the sense that there is a Lipschitz function $\phi^{\prime}$ such that $\phi=\phi^{\prime} m$-a.e. To show this first step we need to assume $\lambda_{u}-\lambda_{s}<\min \left\{\mu_{u}, \mu_{s}\right\}$.

Once we have established this then the proof follows as in the case of uniform contraction, in fact all estimates proceed exactly as in the uniform contraction case. We will only sketch this second part of the proof.

After using a standard re-norming technique to assume without loss of generality that the constants in (1.2), (1.4) are equal to one, it is a straightforward consequence of the definitions of $\lambda_{s}, \lambda_{u}$ that with

$$
K\left(y_{0}\right):=\max _{x \in X}\left\{d\left(g(x) y_{0}, y_{0}\right), d\left(h(x) y_{0}, y_{0}\right)\right\}
$$

we have

$$
d\left(g(x) y, y_{0}\right) \leq e^{\lambda_{u}} d\left(y, y_{0}\right)+K\left(y_{0}\right)
$$

and

Similarly

$$
d\left(h(x) y, y_{0}\right) \leq e^{-\lambda_{s}} d\left(y, y_{0}\right)+K\left(y_{0}\right)
$$

$$
\begin{aligned}
d\left(g(f x) g(x) y, y_{0}\right) & \leq d\left(g(f x) g(x) y, g(f x) y_{0}\right)+d\left(g(f x) y_{0}, y_{0}\right) \\
& \leq e^{\lambda_{u}}\left(e^{\lambda_{u}} d\left(y, y_{0}\right)+K\left(y_{0}\right)\right)+K\left(y_{0}\right) .
\end{aligned}
$$

By induction

$$
d\left(g\left(f^{k} x\right) \cdots g(x) y, y_{0}\right) \leq e^{(k+1) \lambda_{u}} d\left(y, y_{0}\right)+K\left(y_{0}\right)\left(\frac{e^{(k+1) \lambda_{u}}-1}{e^{\lambda_{u}}-1}\right)
$$

and

$$
d\left(h\left(f^{-k} x\right) \cdots h\left(f^{-1} x\right) y, y_{0}\right) \leq e^{-(k+1) \lambda_{s}} d\left(y, y_{0}\right)+K\left(y_{0}\right)\left(\frac{e^{-(k+1) \lambda_{s}}-1}{e^{-\lambda_{s}}-1}\right) .
$$


Let $X_{0}=\{x \in M: \phi(x), \rho(x)$ are defined and $\rho(x) \leq \rho<0\}$. Since $\phi(x)$ is measurable we may choose a set $L \subset M$ and constant $C$ such that $m(L)>\frac{1}{2}$ and for all $x \in L,|\phi(x)| \leq C$. Denote the characteristic function of $L$ by $\xi_{L}$, then by the ergodic theorem for every $x$ in a full measure set $X_{1} \subset M$ the partial sums

$$
\frac{1}{n} \sum_{j=0}^{n-1} \xi_{L}\left(f^{j} x\right) \quad \text { and } \quad \frac{1}{n} \sum_{j=0}^{n-1} \xi_{L}\left(f^{-j} x\right)
$$

converge to $m(L)>\frac{1}{2}$.

Define $X_{2}:=\bigcap_{n \in \mathbb{Z}} f^{n}\left(X_{0} \cap X_{1}\right)$. Note $m\left(X_{2}\right)=1$ and $X_{2}$ is $f$-invariant. As a consequence of the local product decomposition of $m$ [7, Theorem 1(d)] for $m$-a.e. $y$, we have that $m_{s}$-a.e. $x \in W_{\text {loc }}^{u(s)}(y)$ lies in $X_{2}$.

We will, as in the uniform contraction case, prove regularity along local stable/unstable manifolds.

3.1. Regularity on local unstable manifolds. Assume $x, y \in X_{2}$ and $x \in W_{\mathrm{loc}}^{u}(y)$. For all $n \geq 0$ define $x_{n}$ and $y_{n}$ by $x_{n}=f^{-n} x$ and $y_{n}=f^{-n} y$. Then using (1.3) we have

$$
\begin{aligned}
d(\phi(x), \phi(y)) & =d\left(g\left(f^{n-1} x_{n}\right) \cdots g\left(x_{n}\right) \phi\left(x_{n}\right), g\left(f^{n-1} y_{n}\right) \cdots g\left(y_{n}\right) \phi\left(y_{n}\right)\right) \\
& \leq d\left(g\left(f^{n-1} x_{n}\right) \cdots g\left(x_{n}\right) \phi\left(x_{n}\right), g\left(f^{n-1} x_{n}\right) \cdots g\left(x_{n}\right) \phi\left(y_{n}\right)\right)+\sum_{j=0}^{n-1} u_{j}
\end{aligned}
$$

where

$$
\begin{aligned}
u_{j}=d\left(g\left(f^{n-1} x_{n}\right) \cdots\right. & g\left(f^{j+1} x_{n}\right) g\left(f^{j} x_{n}\right) g\left(f^{j-1} y_{n}\right) \cdots g\left(y_{n}\right) \phi\left(y_{n}\right), \\
& \left.g\left(f^{n-1} x_{n}\right) \cdots g\left(f^{j+1} x_{n}\right) g\left(f^{j} y_{n}\right) g\left(f^{j-1} y_{n}\right) \cdots g\left(y_{n}\right) \phi\left(y_{n}\right)\right)
\end{aligned}
$$

(defined appropriately for $u_{0}$ and $u_{n-1}$ ). Let

$$
v_{j}=g\left(f^{j-1} y_{n}\right) \cdots g\left(y_{n}\right) \phi\left(y_{n}\right)=h\left(f^{-(n-j)} y\right) \cdots h\left(f^{-1} y\right) \phi(y) .
$$

Now by Assumptions (I), (II) we have

$$
\begin{aligned}
u_{j} & \leq\left(e^{\lambda_{u}}\right)^{n-j-1} d\left(g\left(f^{j} x_{n}\right) v_{j}, g\left(f^{j} y_{n}\right) v_{j}\right) \\
& \leq\left(e^{\lambda_{u}}\right)^{n-j-1}\left(K_{1}+K_{2} d\left(v_{j}, y_{0}\right)\right) d\left(f^{j} x_{n}, f^{j} y_{n}\right) .
\end{aligned}
$$

Hence

$$
u_{j} \leq C e^{\left(\lambda_{u}-\mu_{u}\right)(n-j)}\left(K_{1}+K_{2} d\left(v_{j}, y_{0}\right)\right) d(x, y)
$$

Furthermore,

$$
\begin{aligned}
d\left(v_{j}, y_{0}\right) & =d\left(h\left(f^{j} y_{n}\right) \cdots h\left(f^{n-1} y_{n}\right) \phi(y), y_{0}\right) \\
& \leq e^{-(n-j+1) \lambda_{s}} d\left(\phi(y), y_{0}\right)+K\left(y_{0}\right)\left(\frac{e^{-(n-j+1) \lambda_{s}}-1}{e^{-\lambda_{s}}-1}\right) .
\end{aligned}
$$

Thus

$$
\begin{aligned}
u_{j} \leq & C\left(K_{1}+K_{3} K\left(y_{0}\right)\right) e^{\left(\lambda_{u}-\mu_{u}\right)(n-j)} d(x, y) \\
& +C K_{4}\left(d\left(\phi(y), y_{0}\right)+K\left(y_{0}\right)\right) e^{\left(-\lambda_{s}+\lambda_{u}-\mu_{u}\right)(n-j)} d(x, y)
\end{aligned}
$$


where $C, K_{i}$ are fixed constants. Hence, under the assumption $\lambda_{u}-\lambda_{s}<\min \left\{\mu_{u}, \mu_{s}\right\}$,

$$
\sum_{j=0}^{n-1} u_{j} \leq \sum_{j=0}^{\infty} u_{j} \leq\left(C^{\prime}+C^{\prime \prime} d\left(\phi(y), y_{0}\right)\right) d(x, y)
$$

for some constants $C^{\prime}, C^{\prime \prime}>0$.

We now consider the first term

$$
u_{* n}:=d\left(g\left(f^{n-1} x_{n}\right) \cdots g\left(x_{n}\right) \phi\left(x_{n}\right), g\left(f^{n-1} x_{n}\right) \cdots g\left(x_{n}\right) \phi\left(y_{n}\right)\right) .
$$

Since $m(L)>\frac{1}{2}$ there is a positive density of times $n$ such that $x_{n} \in L$ and $y_{n} \in L$. At these times $\phi\left(x_{n}\right), \phi\left(y_{n}\right)$ are uniformly bounded by $C$. Since $\rho(x)<\rho$ by assumption of contraction on average, $\lim _{n \rightarrow \infty} u_{* n}=0$.

Thus for $m$-a.e. $y \in M$ and for a.e. $x \in W_{\text {loc }}^{u}(y)$ with respect to the conditional measure $m$ induces on $W_{\text {loc }}^{u}(y)$,

$$
d(\phi(x), \phi(y)) \leq\left(C^{\prime}+C^{\prime \prime} d\left(\phi(y), y_{0}\right)\right) d(x, y) .
$$

3.2. Regularity on local stable manifolds. The argument along local stable manifolds proceeds in the same way with the obvious modifications. We define $x_{n}=f^{n} x, y_{n}=f^{n} y$ and consider the sum

$$
\begin{aligned}
d(\phi(x), \phi(y))= & d\left(h\left(f^{-n} x_{n}\right) \cdots h\left(f^{-1} x_{n}\right) \phi\left(x_{n}\right), h\left(f^{-n} y_{n}\right) \cdots h\left(f^{-1} y_{n}\right) \phi\left(y_{n}\right)\right) \\
\leq & d\left(h\left(f^{-n} x_{n}\right) \cdots h\left(f^{-1} x_{n}\right) \phi\left(x_{n}\right), h\left(f^{-n} x_{n}\right) \cdots h\left(f^{-1} x_{n}\right) \phi\left(y_{n}\right)\right) \\
& +\sum_{j=0}^{n-1} s_{j}
\end{aligned}
$$

where

$$
\begin{array}{r}
s_{j}=d\left(h\left(f^{-n} x_{n}\right) \cdots h\left(f^{-(j+1)} x_{n}\right) h\left(f^{-j} x_{n}\right) h\left(f^{-(j-1)} y_{n}\right) \cdots h\left(f^{-1} y_{n}\right) \phi\left(y_{n}\right),\right. \\
\left.h\left(f^{-n} x_{n}\right) \cdots h\left(f^{-(j+1)} x_{n}\right) h\left(f^{-j} y_{n}\right) h\left(f^{-(j-1)} y_{n}\right) \cdots h\left(f^{-1} y_{n}\right) \phi\left(y_{n}\right)\right) .
\end{array}
$$

The analysis of all terms except the first term

$$
s_{* n}:=d\left(h\left(f^{-n} x_{n}\right) \cdots h\left(f^{-1} x_{n}\right) \phi\left(x_{n}\right), h\left(f^{-n} x_{n}\right) \cdots h\left(f^{-1} x_{n}\right) \phi\left(y_{n}\right)\right)
$$

proceeds as before, using the centre bunching hypothesis $-\lambda_{s}+\lambda_{u}-\mu_{s}<0$.

To deal with the first term (under the assumptions of Theorem 1.12(i)) suppose that $\phi$ is Hölder of exponent $\gamma$ on an open set $S$.

By Poincaré recurrence, almost every point of $\Lambda$ returns to $S$ infinitely often. If $y \in M$ is such a point, then as diam $f^{n} W_{\mathrm{loc}}^{s}(y) \rightarrow 0$ as $n \rightarrow \infty$, we see that there exists an infinite sequence of distinct times $n_{k}$ such that $f^{n_{k}}(x), f^{n_{k}}(y) \in S$. If $f^{n_{k}}(x), f^{n_{k}}(y) \in S$ then $\left|s_{* n_{k}}\right| \leq e^{n_{k}\left(\lambda_{s}-\gamma \mu_{s}\right)}$. By the assumption that $\lambda_{s}-\gamma \mu_{s}<0, \lim _{k \rightarrow \infty}\left|s_{* n_{k}}\right|=0$.

Similarly if $f$ is $s$-conformal and $\left.\phi\right|_{S}$ is Hölder of exponent $\gamma$ where $\mu(S)>0$ then by Lemma A.1 of Appendix A there exists an infinite sequence of distinct times $n_{k}$ such that $f^{n_{k}}(x), f^{n_{k}}(y) \in S$ and hence in this case also $\lim _{k \rightarrow \infty}\left|s_{* n_{k}}\right|=0$. 
Thus for a full $m$-measure set of $y \in M$ and every $x \in W_{\text {loc }}^{s}(y)$ we have

$$
d(\phi(x), \phi(y)) \leq\left(C^{\prime}+C^{\prime \prime} d\left(\phi(y), y_{0}\right)\right) d(x, y) .
$$

Similarly we can show that there exists a full $m$-measure set of $y \in M$ such that for every $x \in W_{\text {loc }}^{u}(y)$ we have

$$
d(\phi(x), \phi(y)) \leq\left(C^{\prime}+C^{\prime \prime} d\left(\phi(y), y_{0}\right)\right) d(x, y) .
$$

The local product structure ensures that a.e. point $y$ has a neighbourhood $U(y)$ containing a ball of uniform diameter, and a constant $K(y)$ such that

$$
d\left(\phi(x), \phi\left(x^{\prime}\right)\right) \leq K(y) d\left(x, x^{\prime}\right)
$$

for $m$-a.e. $x, x^{\prime} \in U(y)$.

By compactness of $M$ this implies that there is a Lipschitz function $\phi^{\prime}(x)$ such that $\phi(x)^{\prime}=\phi(x) m$-a.e.

Once we have established that $\phi$ is Lipschitz if property (i) of Theorem 1.12 does not hold (or property (a) in the case $f$ is $s$-conformal), the proof that $\phi$ is $C^{k-}$ in Theorem 1.12 proceeds exactly as in the case of uniform contraction. The proof that generically condition (i) or (a) does hold also proceeds exactly as in the case of the proof of Theorem 1.3.

Theorem 1.15 is proved by using the arguments of $\$ 3.2$, with obvious modifications, to prove regularity of $\phi$ along local unstable manifolds. This is necessary since we do not have contraction on average which makes the argument establishing regularity along local unstable manifolds easier. In the case that we do not have contraction on average we require the extra condition that $\gamma>\lambda_{u} / \mu_{u}$.

\section{A. Appendix}

Let $m$ be the ergodic invariant measure of maximal entropy for the topologically mixing Axiom A diffeomorphism $f: \Lambda \subset M \rightarrow \Lambda \subset M$.

Let $m_{s}(y)$ (respectively $m_{u}(y)$ ) denote the conditional measure that $m$ induces on $W_{\text {loc }}^{s}(y)$ (respectively $\left.W_{\text {loc }}^{u}(y)\right)$. As a consequence of [7, Theorem 1(c)], for $m$-a.e. $y$ if $A \subset W_{\text {loc }}^{s}(y)$ then

$$
\frac{m_{s}\left(f^{k} y\right)\left(f^{k} A \cap W_{\mathrm{loc}}^{s}\left(f^{k} y\right)\right)}{m_{s}\left(f^{k} y\right)\left(W_{\mathrm{loc}}^{s}\left(f^{k} y\right)\right)}=\frac{m_{s}(y)\left(A \cap W_{\mathrm{loc}}^{s}(y)\right)}{m_{s}(y)\left(W_{\mathrm{loc}}^{s}(y)\right)}
$$

and similarly

$$
\frac{m_{u}\left(f^{-k} y\right)\left(f^{-k} A \cap W_{\mathrm{loc}}^{u}\left(f^{-k} y\right)\right)}{m_{u}\left(f^{-k} y\right)\left(W_{\mathrm{loc}}^{u}\left(f^{-k} y\right)\right)}=\frac{m_{u}(y)\left(A \cap W_{\mathrm{loc}}^{u}(y)\right)}{m_{u}(y)\left(W_{\mathrm{loc}}^{u}(y)\right)} .
$$

LEMMA A.1. Suppose that $m(H)>0$, then if $f$ is $s$-conformal, $m$-a.e. $y$ has the property that for $m_{s}(y)$-a.e. $x \in W_{\mathrm{loc}}^{s}(y)$ there exists an infinite sequence of distinct positive integers $k$ such that $f^{k}(x), f^{k}(y) \in H$.

Proof. By the local product decomposition of $m$ [7, Theorem 1(d)] it follows that $H \cap W_{\text {loc }}^{s}(x)$ has positive $m_{S}(x)$-measure for $m$-a.e. $x \in H$. By [11, Corollary 10.50], 
if $H \cap W_{\mathrm{loc}}^{s}(x)$ has positive $m_{s}(x)$-measure then $m_{s}(x)$-a.e. point of $H \cap W_{\mathrm{loc}}^{s}(x)$ is a point of density for $H$. Thus for $m$-a.e. $z \in H$,

$$
\lim _{\epsilon \rightarrow 0} \frac{m_{s}\left(H \cap W_{\epsilon}^{s}(z)\right)}{m_{S}\left(W_{\epsilon}^{s}(z)\right)}=1
$$

Let $\epsilon_{1}>0$ and define

$$
G_{\epsilon_{1}}^{t}(y):=\left\{z \in W_{\epsilon_{1}}^{s}(y): f^{t}(y), f^{t}(z) \in H\right\} .
$$

Given $\delta>0$ for each $n>1$ there exists a subset $H_{n} \subset H, m\left(H_{n}\right)>0$, and $0<\epsilon_{n}<\epsilon_{1}$ such that

$$
\frac{m_{s}\left(H \cap W_{\epsilon_{n}}^{s}(z)\right)}{m_{S}\left(W_{\epsilon_{n}}^{s}(z)\right)}>1-\frac{\delta}{2^{n}}
$$

for all $z \in H_{n}$ and $0<\epsilon \leq \epsilon_{n}$. Since $H_{n}$ has positive measure, for $m$-a.e. point $z$, $f^{k}(z) \in H_{n}$ for infinitely many $k$. Choose $t_{n}$ so that $d\left(f^{k}(y), f^{k}(z)\right) \leq \epsilon_{n}$ for all $z \in W_{\epsilon_{1}}^{s}(y)$, for all $k>t_{n}$. Choose $r_{n}>t_{n}$ such that $f^{r_{n}}(y) \in H_{n}$. Since the foliation into local stable manifolds is invariant, $f^{r_{n}} W_{\epsilon_{1}}^{s}(y) \subset W_{\text {loc }}^{s}\left(f^{r_{n}}(y)\right)$. Furthermore, since $f$ is $s$-conformal, $f^{r_{n}} W_{\epsilon_{1}}^{s}(y)$ is an open ball (this is where we use the assumption that $f$ is $s$-conformal).

Thus

$$
\frac{m_{s}\left(f^{r_{n}}(y)\right)\left(H \cap f^{r_{n}} W_{\epsilon_{1}}^{s}(y)\right)}{m_{s}\left(f^{r_{n}} y\right)\left(f^{r_{n}} W_{\epsilon_{1}}^{s}(y)\right)}=\frac{m_{s}(y)\left(H \cap W_{\epsilon_{1}}^{s}(y)\right)}{m_{s}(y)\left(W_{\epsilon_{1}}^{s}(y)\right)}>1-\frac{\delta}{2^{n}}
$$

and hence $m_{s}(y)\left(G_{\epsilon_{1}}^{r_{n}}(y) \cap W_{\epsilon_{1}}^{s}(y)\right)>\left(1-\delta / 2^{n}\right) m_{s}(y)\left(W_{\epsilon_{1}}^{s}(y)\right)$.

Thus $m_{s}(y)\left(\cup_{n}\left(G_{\epsilon_{1}}^{r_{n}}(y) \cap W_{\epsilon_{1}}^{s}(y)\right)\right)>1-2 \delta$ and hence $\mu_{s}(y)\left\{z \in W_{\epsilon_{1}}(y): f^{k}(z)\right.$, $f^{k}(y) \in H$ infinitely often for the same values of $\left.k\right\}>1-2 \delta$. Since $\delta$ was arbitrary the lemma is proved.

\section{REFERENCES}

[1] B. Hasselblatt and A. Wilkinson. Prevalence of non-Lipschitz Anosov foliations. Ergod. Th. \& Dynam. Sys. 19 (1999), 643-656.

[2] M. W. Hirsch, C. C. Pugh and M. Shub. Invariant Manifolds (Lecture Notes in Mathematics, 583). Springer, Berlin, 1977.

[3] J.-L. Journé. A regularity lemma for functions of several variables. Revista Matemática Iberoamericana 4 (1988), 187-193.

[4] J. L. Kaplan, J. Mallet-Paret and J. A. Yorke. The Lyapunov dimension of a nowhere differentiable attracting torus. Ergod. Th. \& Dynam. Sys. 4 (1984), 261-281.

[5] V. Niţică and A. Török. Regularity of the coboundary for cohomologous cocycles. Ergod. Th. \& Dynam. Sys. 18 (1998), 1187-1209.

[6] M. Pollicott and C. P. Walkden. The measurable Livšic theorem for connected Lie groups. Trans. Amer. Math. Soc. 353(7) (2001), 2879-2895.

[7] D. Ruelle and D. Sullivan. Currents, flows and diffeomorphisms. Topology 14 (1975), 319-327.

[8] J. Stark. Invariant graphs for forced systems. Physica D 109 (1997), 163-173.

[9] J. Stark. Regularity of invariant graphs for forced systems. Ergod. Th. \& Dynam. Sys. 19 (1999), $155-199$.

[10] C. P. Walkden. The box dimension of invariant graphs over hyperbolic systems. Preprint, 1999.

[11] R. L. Wheeden and A. Zygmund. Measure and Integral: An Introduction to Real Analysis. Marcel Dekker, New York, 1977. 